\title{
Resonant H - Photodetachment: Enhanced Photodestruction and Consequences for Radiative Feedback
}

\section{Citation}

Miyake, S., P. C. Stancil, H. R. Sadeghpour, A. Dalgarno, B. M. McLaughlin, and R. C. Forrey. 2010. "Resonant H - Photodetachment: Enhanced Photodestruction and Consequences for Radiative Feedback." The Astrophysical Journal 709 (2) (January 14): L168-L171. doi:10.1088/2041-8205/709/2//168. http://dx.doi.org/10.1088/2041-8205/709/2/L168.

\section{Published Version}

doi:10.1088/2041-8205/709/2/L168

\section{Permanent link}

http://nrs.harvard.edu/urn-3:HUL.InstRepos:22565794

\section{Terms of Use}

This article was downloaded from Harvard University's DASH repository, and is made available under the terms and conditions applicable to Other Posted Material, as set forth at http:// nrs.harvard.edu/urn-3:HUL.InstRepos:dash.current.terms-of-use\#LAA

\section{Share Your Story}

The Harvard community has made this article openly available.

Please share how this access benefits you. Submit a story.

Accessibility 


\title{
RESONANT $\mathrm{H}^{-}$PHOTODETACHMENT: ENHANCED PHOTODESTRUCTION AND CONSEQUENCES FOR RADIATIVE FEEDBACK
}

\author{
S. Miyake ${ }^{1}$, P. C. Stancil ${ }^{1}$, H. R. Sadeghrour ${ }^{2}$, A. Dalgarno ${ }^{2}$, B. M. Mclaughlin ${ }^{3}$, And R. C. Forrey ${ }^{4}$ \\ ${ }^{1}$ Department of Physics and Astronomy and the Center for Simulational Physics, University of Georgia, Athens, GA 30602-2451, USA; \\ shinyam@physast.uga.edu, stancil@physast.uga.edu \\ 2 ITAMP, Harvard-Smithsonian Center for Astrophysics, Cambridge, MA 02138, USA; hrs@cfa.harvard.edu, adalgarno@cfa.harvard.edu \\ ${ }_{3}^{3}$ David Bates Building, School of Mathematics and Physics, Queen's University Belfast, 7 College Park, Belfast BT7 1NN, UK; b.mclaughlin@qub.ac.uk \\ ${ }^{4}$ Department of Physics, Penn State University, Berks Campus, Reading, PA 19610, USA; rcf6@psu.edu \\ Received 2009 July 31; accepted 2009 December 24; published 2010 January 14
}

\begin{abstract}
The hydrogen negative ion plays a crucial role in the formation of hydrogen molecules in the early universe. Cooling through excitation of $\mathrm{H}_{2}$ drives the formation of the first cosmological objects. The $\mathrm{H}_{2}$ molecules are produced primarily by a reaction sequence initiated by $\mathrm{H}^{-}$. We explore the influence of enhanced photodestruction rates that arise due to absorption by resonance states of $\mathrm{H}^{-}$lying near $11 \mathrm{eV}$. We examine the feedback effects that occur in radiation fields characteristic of Population III stars, blackbody sources, power-law spectra, and the hydrogen Lyman modulated sawtooth spectra of the high-redshift intergalactic medium.
\end{abstract}

Key words: atomic processes - cosmology: theory - early universe - galaxies: formation - galaxies: high-redshift

\section{INTRODUCTION}

After the formation of the first luminous sources, an ultraviolet (UV) background radiation field was built up as the energetic photons traveled through the surrounding primordial gas. The photons ionized the neutral primordial hydrogen and helium atoms formed by recombination, and the universe entered the reionization era (cf. Barkana \& Loeb 1999; Bromm \& Larson 2004). These photons were produced by the first stars (Population III (Pop III) stars), primordial galaxies, and early miniquasars that formed from halos of gas collapsing under the action of gravitational forces between baryons and dark matter. The gravitational energy resulting from the adiabatic collapse was dissipated by radiative cooling processes. The primary cooling agent for temperatures below $8000 \mathrm{~K}$ was molecular hydrogen (Saslaw \& Zipoy 1967; Peebles \& Dicke 1968). $\mathrm{H}_{2}$, therefore, played a critical role in the evolution of primordial halos and the subsequent formation of the first generation of luminous sources.

The UV photons from these sources ionized the intergalactic medium (IGM) and modified the chemistry that created and destroyed the hydrogen molecules. Feedback mechanisms, enhancing and suppressing the efficiency of subsequent star formation, may have occurred (Haiman et al. 1996, 1997, 2000; Kitayama et al. 2001; Yoshida et al. 2003).

Molecular hydrogen was formed in the early universe by the $\mathrm{H}^{-}$reaction sequence

$$
\begin{aligned}
\mathrm{H}+e^{-} & \rightarrow \mathrm{H}^{-}+v, \\
\mathrm{H}^{-}+\mathrm{H} & \rightarrow \mathrm{H}_{2}+e^{-},
\end{aligned}
$$

in which electrons behaved as catalysts. The process of associative detachment (Equation (2)) was introduced by Dalgarno (cf. Pagel 1959) and McDowell (1962) pointed to it as a source of $\mathrm{H}_{2}$ in the interstellar medium. The electrons produced by photoionization increase the production of $\mathrm{H}_{2}$ and constitute a positive feedback. A negative feedback arises from the photodissociation of $\mathrm{H}_{2}$ by UV radiation between the Lyman limit $(13.6 \mathrm{eV})$ and the threshold for absorption in the Lyman and Werner systems of
$\mathrm{H}_{2}(11.18 \mathrm{eV})$, followed by spontaneous radiative decay into the vibrational continuum. The negative feedback may therefore be amplified by the photodetachment of $\mathrm{H}^{-}$(Chuzhoy et al. 2007; Glover 2007),

$$
\mathrm{H}^{-}+v \rightarrow \mathrm{H}+e^{-} .
$$

Chuzhoy et al. (2007) estimated the magnitude of a radiative feedback effect due to the photodetachment of $\mathrm{H}^{-}$by introducing a suppression factor

$$
F_{b}=1+\frac{\beta_{3}}{k_{2} n_{\mathrm{H}}}
$$

where $\beta_{3}$ is the $\mathrm{H}^{-}$photodetachment rate for a given radiation field, $k_{2}$ is the rate coefficient for associative detachment (Reaction (2)), and $n_{\mathrm{H}}$ is the number density of atomic hydrogen. The abundance of $\mathrm{H}^{-}$is thereby reduced by $F_{\mathrm{b}}$, hence suppressing the production of $\mathrm{H}_{2}$. This expression ignores the effect of the removal of $\mathrm{H}^{-}$by mutual neutralization (Dalgarno \& McCray 1973):

$$
\mathrm{H}^{+}+\mathrm{H}^{-} \rightarrow \mathrm{H}+\mathrm{H}
$$

In their analysis, Chuzhoy et al. (2007) employed the commonly adopted $\mathrm{H}^{-}$photodetachment cross section, from a fit to the results of Wishart (1979), which includes only non-resonant contributions as shown in Figure $1 . \mathrm{H}^{-}$has two discrete levels: the ground state is ${ }^{1} S_{e}$, while the other bound level has ${ }^{3} P_{e}$ symmetry whose decay to the ground state is spin forbidden (Bylicki \& Bednarz 2000). As such $\mathrm{H}^{-}$has no discrete spectrum, but in the continuum region, $\mathrm{H}^{-}$has a strong series of broad and narrow auto-detaching resonances, for photon energies greater than $\sim 11$ eV (Rau 2002; Stancil et al. 2010).

We have revisited the photodetachment of $\mathrm{H}^{-}$and obtained accurate cross sections based on a hybrid approach of $R$-matrix calculations, incorporation of high-resolution crosssection measurements, and previous theoretical results (B. M. McLaughlin et al. 2010, in preparation). We then constructed a photodetachment cross section, displayed in Figure 1, which is 


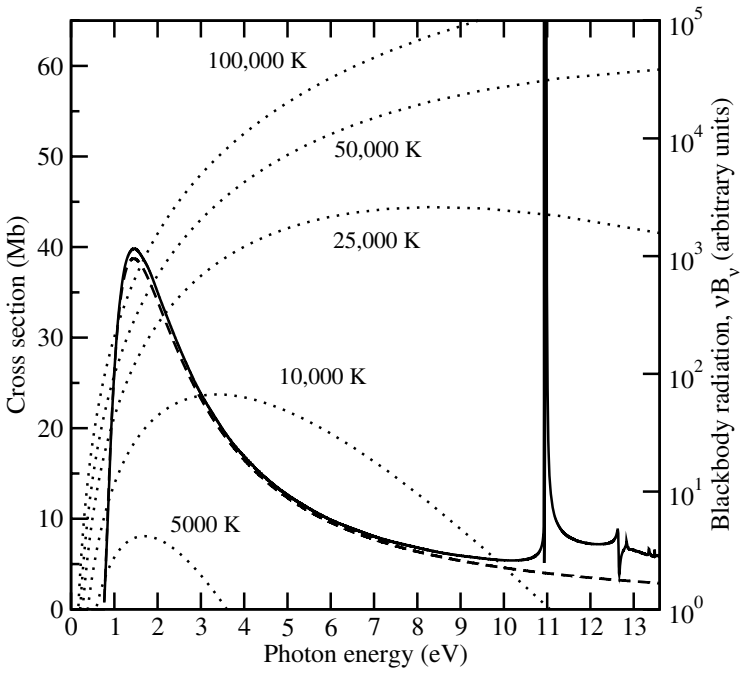

Figure 1. $\mathrm{H}^{-}$photodetachment cross sections. Background without resonances from Chuzhoy et al. (2007) fits to Wishart (1979; dashed); with resonances from B. M. McLaughlin et al. (2010, in preparation; solid). The shape resonance just above $\mathrm{H}(n=2)$ is prominent at about $11 \mathrm{eV}$. A narrow Feshbach resonance is evident below $\mathrm{H}(n=2)$, and there are other less-prominent Feshbach resonances below $\mathrm{H}(n=3)$ near $12.8 \mathrm{eV}$. Also shown are blackbody spectra for temperatures from 5000 to $100,000 \mathrm{~K}$ (dotted).

obtained after a number of oscillator strength sum rules are selfconsistently satisfied (B. M. McLaughlin et al. 2010, in preparation). The new cross section is in good agreement with the results of the eigenchannel $R$-matrix study of the auto-detaching resonances by Sadeghpour et al. (1992), and use of the latter would not change the conclusions presented below.

In this Letter, we point out that the $\mathrm{H}^{-}$auto-detaching resonances provide an additional contribution to the radiative feedback considered by Chuzhoy et al. (2007), which should be included in models of high-redshift halo evolution. In the following section, we estimate the resonant contribution in three high-redshift scenarios that are relevant to the reionization epoch following the formation of the first stars.

\section{RESONANT PHOTODESTRUCTION}

To estimate the effect of the auto-detaching resonant contribution to radiative feedback, we partition photodetachment into the continuum background $(b)$ and resonant $(r)$ contributions. If the rate due to the background is $\beta_{3}^{b}$ and that due to the full cross section is $\beta_{3}^{r+b}$, their ratio $R$ gives the enhancement factor due to the resonances only

$$
R=\frac{\beta_{3}^{r+b}}{\beta_{3}^{b}} .
$$

Similarly, a ratio of $\mathrm{H}_{2}$ suppression factors can be written

$$
F_{r}=\frac{F_{r+b}}{F_{b}}=\frac{1+\frac{\beta_{3}^{r+b}}{n_{\mathrm{H}} k_{2}}}{1+\frac{\beta_{3}^{b}}{n_{\mathrm{H}} k_{2}}},
$$

where $F_{r}$ is the resonant contribution to $\mathrm{H}_{2}$ suppression. Here we have followed the approach of Chuzhoy et al. (2007) and neglected the mutual neutralization process (Equation (5)). For any meaningful suppression of the rate of formation of $\mathrm{H}_{2}$, we would expect the ratio $\beta_{3} / n_{\mathrm{H}} k_{2} \gg 1$, so that

$$
F_{r} \approx \frac{\beta_{3}^{r+b}}{\beta_{3}^{b}}=R .
$$

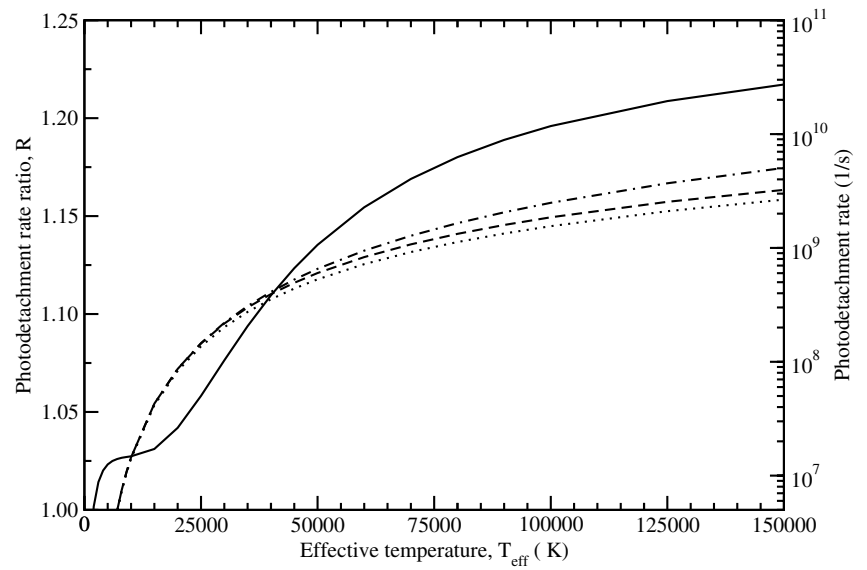

Figure 2. Photodetachment rate ratio, $R$, for a blackbody radiation field as a function of effective temperature $T_{\text {eff }}$ (solid). As discussed in the text $R \sim F_{r}$, the enhancement factor due to the resonances. Also shown are the photodetachment rates: using the Wishart (1979) cross section with a cutoff energy of $13.6 \mathrm{eV}$ (dotted), using the current cross section (B. M. McLaughlin et al. 2010, in preparation) with the cutoff (dashed), and using B. M. McLaughlin et al. (2010, in preparation) without the cutoff, i.e., a full blackbody (dot-dash).

This is the case if fiducial values of the ionization fraction $(x)$ and the fraction of escaping ionizing photons $\left(f_{\text {esc }}\right)$ are adopted as in Equation (10) of Chuzhoy et al. (2007). Therefore, the total $\mathrm{H}_{2}$ suppression factor is given by $F=F_{b} \times F_{r}$, within the approximations discussed above.

\subsection{Pop III Radiation and Recombination}

Radiation from Pop III stars will photoionize the primordial gas in halos in which the stars are formed, and in the gas beyond. The resulting protons recombine producing a spectrum whose photons have energies less than $10.25 \mathrm{eV}$ (case $\mathrm{B}$ recombination for $\mathrm{H}$ ), but greater than $0.755 \mathrm{eV}$. The negative feedback proposed by Chuzhoy et al. (2007) is the photodetachment of $\mathrm{H}^{-}$by the recombination photons, yielding a suppression factor of $F_{b} \sim 800$ (for $x=0.1, f_{\text {esc }}=0.1$ ). The Pop III stars also produce a blackbody continuum with a significant flux of photons less than the Lyman limit $(13.6 \mathrm{eV})$. Depending on the mass of the Pop III star, inclusion of the blackbody spectrum as a source of $\mathrm{H}^{-}$photodetachment could increase the suppression by factors of 1.1 to $\sim 10$ (see Figure 2 in Chuzhoy et al.), increasing as the stellar effective temperature $T_{\text {eff }}$ decreases. The suppression factor arising from the blackbody continuum is enhanced compared to that due to the recombination photons because as $T_{\text {eff }}$ is decreased, fewer photons contribute to $\mathrm{H}$ photoionization, but the Planck spectrum overlap with the $\mathrm{H}^{-}$ photodetachment cross-section peak, near $1.5 \mathrm{eV}$, is enhanced as illustrated in Figure 1.

The enhancement factor $R$ for resonant photodetachment is shown in Figure 2 as a function of the Pop III stellar effective temperature where only photon energies less than the Lyman limit are included. The resonant contribution is seen to increase the $\mathrm{H}_{2}$ suppression factor $F_{r}$ for $T_{\text {eff }}>1000 \mathrm{~K}$ reaching asymptotically $R \sim F_{r} \sim 1.2$ as $T_{\text {eff }}$ exceeds $100,000 \mathrm{~K} .{ }^{5}$ The behavior of $R$ can be understood by comparing the blackbody spectrum to the photodetachment cross sections

\footnotetext{
5 The shoulder between 5000 and $15,000 \mathrm{~K}$ is actually due to the $3 \%$ difference between the two cross sections at the peak near $1.5 \mathrm{eV}$. The fit given by Chuzhoy et al. (2007) does not accurately reproduce the Wishart (1979)
} data at these photon energies. 


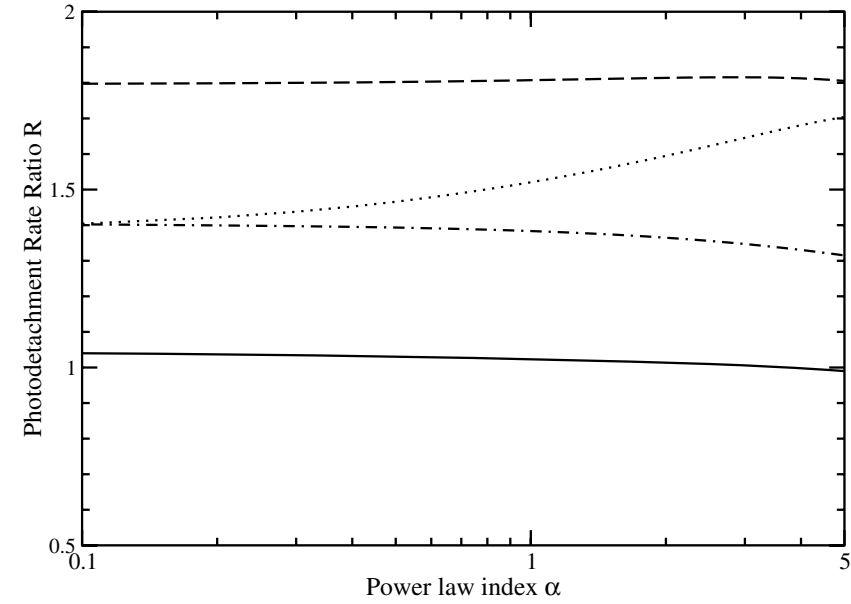

Figure 3. Photodetachment rate ratio, $R$, for a power-law radiation field as a function of the power-law index $\alpha$. Power-law rates computed over the photon energy ranges: $0.75-1000 \mathrm{eV}$ (solid), $10-13.6 \mathrm{eV}$ (dashed), and 10-1000 eV (dotted). The photodetachment rate ratio is also shown for the IGM spectrum of Figure 4 for photon energies 10-13.6 eV (dot-dash).

as shown in Figure 1. As $T_{\text {eff }}$ increases, a larger fraction of photons reside in the region of the resonances compared to the photodetachment cross-section peak near $1.5 \mathrm{eV}$. For example, the blackbody radiation field enhances the suppression factor to $F_{b} \sim 1600$ at 40,000 K (Chuzhoy et al. 2007) with the resonant contribution increasing this by another $20 \%$.

Figure 2 displays the $\mathrm{H}^{-}$photodetachment rates corresponding to the cross section of B. M. McLaughlin et al. (2010, in preparation), which includes the resonant enhancement, with and without the Lyman limit cutoff. Comparison is made to the rate computed with the Lyman limit cutoff using the fit to the non-resonant cross section of Wishart (1979). The differences in the rates are negligible for $T_{\text {eff }}<25,000 \mathrm{~K}$, but increase with increasing $T_{\text {eff }}$.

\subsection{A Smooth Power-law UV Spectrum}

On cosmological scales, the accumulated UV spectrum due to a large number of sources, or to an early miniquasar, is typically represented by a simple power law of the form

$$
J\left(E_{\mathrm{ph}}\right)=J_{21}\left(\frac{E_{\mathrm{ph}}}{E_{\mathrm{H}}}\right)^{-\alpha},
$$

where $J_{21}$ is the intensity in $10^{-21} \mathrm{erg} \mathrm{cm}^{-2} \mathrm{~Hz}^{-1} \mathrm{sr}^{-1}, E_{\mathrm{ph}}$ is the photon energy, $E_{\mathrm{H}}$ is the ionization energy of $\mathrm{H}$, and $\alpha$ is the power-law index. For $\alpha=1.7$, typical of a miniquasar, Chuzhoy et al. (2007) found that the $\mathrm{H}_{2}$ suppression is enhanced by a factor of $\sim 5$ over that due to recombination photons, assuming that the power-law field extends down to the $\mathrm{H}^{-}$ photodetachment threshold of $0.755 \mathrm{eV}$.

Enhancements to the photodetachment rate due to the autodetaching resonances are shown in Figure 3, where we have considered $\alpha=0.1-5$ and various photon energy windows. In addition to miniquasars with $\alpha \sim 1.7$, typical power-law indices for quasars, massive black holes, and the high-redshift IGM are $\sim 0.5-0.7, \sim 1$, and $\sim 0.7-1$, respectively. For the largest range in photon energies $(0.75-1000 \mathrm{eV})$, the enhancement is seen to be small approaching only $4 \%$ for the flattest spectra. However, it might be unrealistic to assume that the radiation intensity extends down to the $\mathrm{H}^{-}$threshold for very steep spectral indices or for sources that emit primarily in the UV. Some authors only considered photon energies greater than $13.6 \mathrm{eV}$

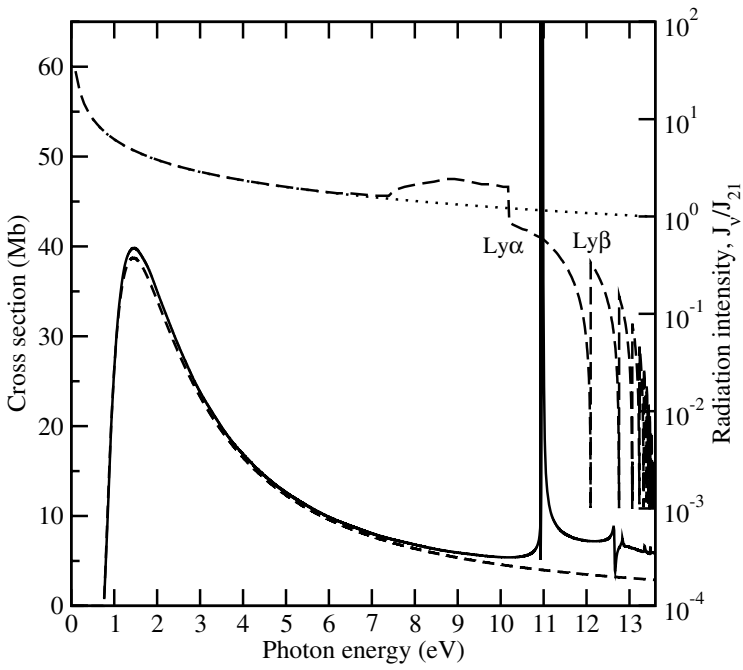

Figure 4. IGM sawtooth spectrum at $z \sim 20$ (long dashed) compared to an $\alpha=0.7$ power-law spectrum (dotted) and $\mathrm{H}^{-}$photodetachment cross sections (same as Figure 1). See the text for a description of the sawtooth spectrum. Note the $\mathrm{H}^{-}$resonance between the Ly $\alpha$ and $\operatorname{Ly} \beta$ features of the IGM spectrum.

for photoionization models due to quasar spectra (Donahue \& Shull 1991; Thoul \& Weinberg 1996) or greater than the LymanWerner band threshold $(11.18 \mathrm{eV})$ when modeling radiative feedback (Haiman et al. 2000). To include the $\mathrm{H}^{-}$resonant and Ly $\alpha$ energies, we computed photodestruction rates for $E_{\mathrm{ph}}>10$ $\mathrm{eV}$ with and without the Lyman limit cutoff which resulted in significant resonant enhancement factors of $R \sim 1.8$ and 1.4-1.7, respectively. With a Lyman limit cutoff, the photodetachment rate integral is constrained to a narrow photon energy bin and is therefore relatively insensitive to the power-law index. Conversely, without a Lyman limit cutoff, the photodetachment rate integral can sample the higher-energy cross section away from the resonances which reduces the enhancement factor for smaller $\alpha$. However, as $\alpha$ is increased $R$ approaches the Lymanlimit value, as the steeper spectral profile favors photon energies near the resonances.

\subsection{IGM Background with H Sawtooth Absorption Modulation}

While the use of power-law spectra is computationally convenient, a fraction of the UV photons will be removed from the IGM spectrum as radiation is transported through the partially neutral IGM. As in the interstellar medium of the Galaxy, photoionization of $\mathrm{H}$ will remove nearly all the photons with energies greater than the Lyman limit, and redshifted resonant Lyman lines will carve out flux from the IGM spectrum. We assume that all sources turn on simultaneously at some redshift $z$, the sources are distributed uniformly in space, and the emission spectrum is given by a power law (Haiman et al. 1997).

To construct a complete IGM spectrum to cover photon energies accessible for $\mathrm{H}^{-}$photodetachment, we combined the $z \sim 20$ spectra presented in Yoshida et al. (2003) from $11-13.6 \mathrm{eV}$ and that given in Haiman et al. (1997) from $6-11 \mathrm{eV}$ with the $\alpha=0.7$ power law from $0.75-6 \mathrm{eV}$. For other power laws, we appropriately scaled the full hybrid spectrum. The resulting $\alpha=0.7$ sawtooth spectrum is shown in Figure 4, with the $\alpha=0.7$ power-law spectrum shown for reference. The two $\mathrm{H}^{-}$photodetachment cross sections are also shown. Interestingly, the $\mathrm{H}^{-}$resonance lies in the window between the Ly $\alpha$ and $\operatorname{Ly} \beta$ IGM spectral features. Because the powerlaw and IGM spectra are similar over most of the photon 
energy range, the resonant enhancement is expected to be comparable for the two cases. In fact, $R$ for the IGM spectrum for $0.75-13.6 \mathrm{eV}$ is nearly identical to the enhancement computed for the $0.75-1000 \mathrm{eV}$ power-law spectrum shown in Figure 3 giving only a modest $4 \%$ enhancement for the smallest powerlaw index. However, if we truncate the IGM photon energy range to $10-13.6 \mathrm{eV}$, the enhancement factor varies slowly between 1.3 and 1.4, somewhat less than the comparable smooth power-law cases, but still significant.

\section{CONCLUSIONS}

Cosmology is now a precision science and if the observations of the early universe are to yield a comprehensive view of its evolution, an accurate detailed account of the atomic and molecular processes that occurred must be constructed. Following the formation of the first stars, Pop III stars, which are assumed to be massive, hot, and luminous, the subsequent role of their radiation on their host primordial halos and the surrounding IGM likely had a significant impact on subsequent primordial star formation. Whether this radiative feedback effect was positive or negative, i.e., enhancing or suppressing the efficiency of star formation, is not fully understood and therefore a topic of considerable current interest.

It has been suggested by Chuzhoy et al. (2007) that $\mathrm{H}^{-}$photodetachment contributes a significant enhancement to a negative feedback effect. We point out in this Letter that the $\mathrm{H}^{-}$ auto-detaching resonances, which occur for photon energies near $11 \mathrm{eV}$, can provide an additional negative feedback contribution. While the resonant contribution is not large for steep power-law spectra extending into the near infrared, it is significant for blackbody spectra with effective temperatures exceeding $\sim 40,000 \mathrm{~K}$ and for the UV background spectrum of the high-redshift IGM. If the background radiation field, after the formation of the first luminous objects, was dominated by miniquasars, or similar sources, there may have been a significant negative radiative feedback effect on the creation of $\mathrm{H}_{2}$, its cooling efficiency, and on the ability of the residual primordial gas to coalesce into another generation of primordial stars. ${ }^{6}$ Therefore $\mathrm{H}^{-}$photodetachment and its auto-detaching resonances should be accurately treated in radiation-hydrodynamic simulations of primordial halos, particularly in the early reionization epoch.

S.M. and P.C.S. acknowledge support from NSF Grant AST0607733, while A.D. acknowledges NSF Grant AST-0607532. The work of B.M.McL. and R.C.F. was supported by a research development grant from the Pennsylvania State University and NSF grant No. PHY-0554794. P.C.S., B.M.McL., and R.C.F thank the Institute for Theoretical Atomic, Molecular, and Optical Physics at the Harvard-Smithsonian Center for Astrophysics for travel support.

\section{REFERENCES}

Barkana, R., \& Loeb, A. 1999, ApJ, 523, 54

Bromm, V., \& Larson, R. B. 2004, ARA\&A, 42, 1

Bylicki, M., \& Bednarz, E. 2000, Phys. Rev. A, 67, 022503

Chuzhoy, L., Kuhlen, M., \& Shapiro, P. R. 2007, ApJ, 665, L85

Dalgarno, A., \& McCray, R. 1973, ApJ, 180, 95

Donahue, M., \& Shull, J. M. 1991, ApJ, 383, 511

Glover, S. C. O. 2007, MNRAS, 379, 1352

Haiman, Z., Abel, T., \& Rees, M. J. 2000, ApJ, 534, 11

Haiman, Z., Rees, M. J., \& Loeb, A. 1996, ApJ, 467, 522

Haiman, Z., Rees, M. J., \& Loeb, A. 1997, ApJ, 476, 458

Kitayama, T., Susa, H., Umemura, M., \& Ikeuchi, S. 2001, MNRAS, 326 1353

McDowell, M. R. C. 1962, Observatory, 81, 240

Pagel, B. E. J. 1959, MNRAS, 119, 609

Peebles, P. J. E., \& Dicke, R. H. 1968, ApJ, 154, 891

Rau, A. R. P. 2002, Astronomy-inspired Atomic and Molecular Physics, Astrophys. Space Sci. Lib., Vol. 271 (Dordrecht: Kluwer)

Sadeghpour, H. R., Greene, C. H., \& Cavagnero, M. 1992, Phys. Rev. A, 45, 1587

Saslaw, W. C., \& Zipoy, D. 1967, Nature, 216, 976

Stancil, P. C., Miyake, S., Sadeghpour, H. R., McLaughlin, B. M., \& Forrey, R. C. 2010, in Proc. Dalgarno Celebratory Symp. (London: Imperial College Press), 102

Thoul, A. A., \& Weinberg, D. H. 1996, ApJ, 465, 608

Wishart, A. W. 1979, MNRAS, 187, 59

Yoshida, N., Abel, T., Hernquist, L., \& Sugiyama, N. 2003, ApJ, 592, 645
6 Note that since the $\mathrm{H}^{-}$mechanism for forming $\mathrm{H}_{2}$ is suppressed, other routes such as the charge exchange reaction $\mathrm{H}+\mathrm{H}_{2}^{+} \rightarrow \mathrm{H}_{2}+\mathrm{H}^{+}$would become important. However, the destruction of $\mathrm{H}_{2}^{+}$due to photodissociation via a far UV radiation field would also contribute to a negative feedback effect. We do not explore these effects here. 